Clio. Femmes, Genre, Histoire

8 | 1998

Georges Duby et l'histoire des femmes

\title{
Des poissons difficiles à pêcher (Italie)
}

\section{Maria-Giuseppina MUZZARELLI}

\section{(2) OpenEdition}

\section{Journals}

Édition électronique

URL : https://journals.openedition.org/clio/316

DOI : 10.4000/clio.316

ISSN : 1777-5299

Éditeur

Belin

Édition imprimée

Date de publication : 1 novembre 1998

ISBN : 2-85816-379-0

ISSN : $1252-7017$

\section{Référence électronique}

Maria-Giuseppina MUZZARELLI, «Des poissons difficiles à pêcher (Italie) », Clio. Histoire, femmes et sociétés [En ligne], 8| 1998, mis en ligne le 03 juin 2005, consulté le 23 avril 2022. URL : http:// journals.openedition.org/clio/316 ; DOI : https://doi.org/10.4000/clio.316

Ce document a été généré automatiquement le 23 avril 2022.

Tous droits réservés 


\title{
Des poissons difficiles à pêcher (Italie)
}

\author{
Maria-Giuseppina MUZZARELLI
}

1 Au moment d'assigner un terminus a quo à cette réflexion sur les rapports entre l'historiographie italienne des femmes et Georges Duby, il me revient en mémoire le célèbre sonnet où Pétrarque évoque l'instant où il tomba amoureux : "Benedetto sia il giorno... l'ora e 'l punto» (Canzoniere, LXI). «Le point », donc: le problème est le point d'où partir.

Duby nous offre un repère, lorsqu'il écrit à la fin de l'un de ses derniers livres :

3 Il y a quinze ans, dans la dernière phrase d'un livre, Le Chevalier, la Femme et le Prêtre, je posai la question : que savons-nous des femmes? Depuis j'ai cherché, parmi toutes les traces qu'ont laissées les dames du XII ${ }^{\mathrm{e}}$ siècle $^{1}$.

4 Qui n'a lu ce livre, s'il aime le Moyen Age $^{2}$ ? Mais parcourons-en la table des matières, nous verrons que le mot «femme » évoqué par le titre en est quasi absent, alors que sa présence traverse tout l'ouvrage.

5 J'ignore s'il est justifié de parler d'un « effet Duby » sur l'histoire des femmes, en particulier en Italie. Mais, s'il est avéré, il aura consisté à rendre explicite ceci, que l'historiographie sous-estimait ou donnait pour acquis, à savoir que les femmes aussi ont fait le Moyen Âge, bien que les traces qu'elles ont laissées soient à peine perceptibles. Dans le livre évoqué, Duby traitait de rois, de princes, de chevaliers et de prêtres, et pourtant chaque page ou presque laisse deviner la présence des femmes. Autrement dit, Georges Duby a su en retrouver les traces lorsqu'il a reconstitué l'histoire de seigneurs et d'évêques, à une époque où faire l'histoire des femmes revenait à la mettre à part, hors de l'histoire générale. Une histoire des femmes sui generis, dira-t-on. Il s'est agi, en réalité, non pas d'écrire une histoire des femmes au sens propre, mais d'élargir les perspectives de manière à les y faire entrer en grand nombre, de dédouaner en quelque sorte les thématiques féminines pour les accueillir dans le cercle de la grande histoire - celui-là même où se mouvait Duby - et de diffuser aussi ces thèmes dans le grand public, un public que les questions concernant le Moyen 
Age commençaient à séduire, il y a de cela tout juste vingt ans, grâce en particulier au talent narratif exceptionnel de notre auteur.

6 À preuve de cet "effet Duby", l'élargissement de la perspective me semble bien exprimé, dans l'édition italienne, par la foule de titres consacrés au Moyen Âge et aux thèmes féminins qui ont précisément suivi la publication de Le Chevalier, la femme et le prêtre. Il suffit, pour le vérifier, de parcourir le catalogue des Éditions Laterza, l'un des éditeurs italiens les plus riches en titres dans ce domaine. Certes, le phénomène peut être un effet de mode, mais, dans ce cas, G. Duby en serait l'un des promoteurs, certainement pas un épigone.

7 Le Chevalier, la femme et le prêtre aurait donc tous les titres à être pris comme terminus a quo, comme « le point» dont parlait Pétrarque. Je partirai pourtant plutôt de 1977, quand G. Duby participa à la Semaine d'études de Spolète consacrée, cette année-là, au mariage dans la société du haut Moyen Âge. Le mariage est, du reste, un thème assez central dans Le Chevalier, la femme et le prêtre pour que le livre porte en sous-titre Le mariage dans la France féodale. C'est aussi un thème récurrent dans toute la production de G. Duby relative aux femmes ${ }^{3}$. On verra que c'est enfin un domaine où l'historiographie italienne attentive aux rapports de sexes s'est montrée particulièrement active.

8 À Spolète, G. Duby tint le discours d'ouverture ${ }^{4}$. Il n'y dit pas grand-chose sur les femmes, et il est clair que les interventions qui ont suivi n'ont pu subir son influence. Mais ses mises en garde contre deux erreurs de perspective où il est facile de tomber quand on étudie le mariage, comportaient deux recommandations importantes pour ceux qui à l'époque s'occupaient d'histoire des femmes. La première était de ne pas accorder de valeur excessive aux normes. La seconde invitait à ne pas adopter sans de nécessaires précautions le point de vue des hommes d'Église ${ }^{5}$. G. Duby recommandait aussi de tirer parti d'autres sources que juridiques et ecclésiastiques, non seulement pour étudier les conceptions et la pratique du mariage, mais pour discuter l'idée que les laïcs se faisaient des femmes et la condition sociale réelle de celles-ci.

9 Ces règles ainsi énoncées dès 1976, G. Duby les a par la suite appliquées à l'histoire des femmes, choisissant le XII ${ }^{e}$ siècle pour sonder le plus grand nombre possible de sources et retracer des profils de femmes qui ne fussent pas des marginales.

\section{Femmes remarquables ou situations exceptionnelles?}

10 Vito Fumagalli est de ceux qui, en Italie, dans un parcours de recherche comparable à celui de G. Duby, suivaient alors les traces de personnages féminins remarquables. Dans une note bibliographique concluant le livre qu'il a consacré à la comtesse Mathilde de Canossa, il écrit : "Sur la femme et la conception qu'on en a au Moyen Age, il suffit de renvoyer à G. Duby et à son Donne nello specchio del Medioevo $0^{6}$ ». Ce n'est pas là une banale référence bibliographique, mais la déclaration d'une réelle convergence de vues. Nous la saisissons bien dans le travail par lequel V. Fumagalli a reconstruit l'histoire et le profil de Mathilde de Canossa ou d'Adélaïde de Turin ${ }^{7}$. Pour reprendre la définition de Benzone d'Alba, Adélaïde était « un poisson impossible à pêcher ». Belle et efficace formule, qui m'a semblé la mieux à même d'intituler cet essai sur l'historiographie consacrée aux femmes dans la période étudiée par G. Duby ${ }^{8}$. Mathilde et Adélaïde étaient deux femmes nobles et puissantes, comme l'était l'Aliénor d'Aquitaine étudiée par G. Duby, et elles ont influé de manière décisive sur les événements politiques de 
leur temps. On aurait bien pu parler de Mathilde comme d'une reine, ou plutôt d'un roi, selon G. M. Cantarella, car les qualités de la comtesse étaient éminemment masculines ${ }^{9}$.

11 On le sait, la célébration des mérites de quelques femmes d'exception relève d'une tradition historiographique très ancienne : le genre en a été inauguré par Plutarque. Diane 0 . Hughes a bien noté que ces femmes sont généralement présentées sous une forme iconique, c'est-à-dire plus exemplaire que narrative ${ }^{10}$, et Mathilde constitue d'ordinaire le plus beau fleuron de cette couronne de grandes dames ${ }^{11}$. Pour sortir du cadre traditionnel des louanges concernant de rares héroïnes, l'historiographie récente s'est tournée vers des personnages au profil plus modeste, des femmes travaillant durement sans tirer de leur peine satisfaction ni reconnaissance sociale, des femmes obscures, en somme, pour découvrir en fin de compte qu'il ne s'agissait pas à proprement parler de femmes vaincues.

Quand sortit en 1989 le recueil d'essais Medioevo al femminile, Ferruccio Bertini, qui l'avait dirigé, s'empressa de déclarer que les protagonistes féminins de cet ouvrage s'y côtoyaient en raison d'une particularité commune, jusque-là négligée : elles n'étaient puissantes ni par la naissance ni par leur mariage, mais elles avaient laissé d'ellesmêmes des traces directes dans leurs œuvres écrites ${ }^{12}$. Il ne s'agissait donc pas de ces quelques femmes d'exception couramment privilégiées par les historiens des siècles passés. Égérie, Baudonivia, Dhuoda, Roswitha, Trotula, Héloïse, Hildegarde et Catherine avaient pris la plume et laissé un témoignage écrit ou personnel de leurs pensées ${ }^{13}$. G. Duby, de son côté, clôt le volume médiéval de l'Histoire des femmes en Occident en soulignant que les femmes parlaient, et même beaucoup, au Moyen Âge ${ }^{14}$. F. Bertini nous rappelle, pour sa part, qu'elles écrivaient. Pour approcher quelques-unes d'entre elles il les a lues, et cette opération, nous dit-il, « réserve des surprises extraordinaires, et révèle des ouvertures inattendues et insoupçonnées ${ }^{15}$ ». Ce type d'entreprise jette un pont entre la tradition historiographique et des exigences nouvelles. Les volumes qui ont suivi dans la même collection et qui sont consacrés à la Renaissance et à l'âge baroque ont encore accentué ce dessein en proposant des biographies de femmes «le moins possible "au-delà de leur sexe"; des personnages moyens, en quelque sorte, tirant leur exemplarité [...] de divers aspects de l'histoire générale de leur temps, et éventuellement situés dans les engrenages de l'histoire, non pas au-dessus d'eux ${ }^{16} »$.

Si l'une des tendances de l'historiographie italienne des quinze dernières années a précisément consisté à approcher des femmes quelconques par leurs biographies, Duby n'a rien à voir avec cette orientation de recherche. Au contraire, c'est justement dans les années où ce processus se dessinait qu'il a fait un retour critique à la tradition en s'appuyant, entre autres, sur sa capacité à résoudre cas et problèmes par un récit constituant presque un unicum.

14 En Italie, Vito Fumagalli a suivi un chemin parallèle. Revenons à son essai Adelaide $e$ Matilde. De ces deux femmes, Fumagalli n'a pas voulu suggérer l'exceptionnalité personnelle. Pour des raisons diverses, elle est certes évidente, mais elle est aussi trompeuse, puisque c'est à leur famille que ces femmes devaient leur rôle historique. Fumagalli voulait plutôt inscrire leur exceptionnalité dans celle du moment où elles vivaient ${ }^{17}$. Le rôle historique de ces deux femmes reflèterait donc une situation générale assez singulière pour qu'on puisse définir le XIe siècle comme un "siècle ouvert aussi aux femmes ». Le pouvoir féminin de Mathilde ou d'Adélaïde s'affirme au moment même où les structures de l'État carolingien se désagrègent, où l'unité de l'Empire éclate, et où un bouillonnement de forces suscite particularismes et 
antagonismes, éveille volontés et velléités. La réalité, qui auparavant se trouvait en quelque sorte coordonnée et soumise à un contrôle supérieur, est alors remise en jeu. « Adélaïde de Turin et Mathilde de Canossa se situent à la sortie d'une telle crise ${ }^{18}$ ». Toutes deux sont à la tête d'une marche importante, toutes deux contrôlent des points stratégiques et toutes deux luttent contre la précarité, le particularisme et la fragmentation politiques. Toutes deux, enfin, ont vécu dans une phase historique où tout était possible, le rôle exceptionnel, en particulier, qu'elles ont tenu dans un scénario. Et ce scénario explique leur rôle, dans la mesure où la charge qu'elles ont assumée était assez importante pour faire passer au second plan le fait que la personne la revêtant était une femme. Telle est la principale nouveauté de la lecture faite par V. Fumagalli.

15 Ni chez V. Fumagalli ni chez M. Oldoni, pour en rester à ces deux historiens, ne pointe l'envie de parler de femmes ayant surpassé leur sexe ${ }^{19}$; mais leur intérêt le plus vif les a portés vers une période historique qui a autorisé de tels surpassements en grand nombre, $\mathrm{y}$ compris ceux qui brisaient parfois les barrières de sexe - comme le montrent Angelberga, femme de Louis II, Ageltrude, l'épouse de Gui de Spolète, ou Berthe, celle d'Adalbert II de Tuscie ${ }^{20}$.

La période qui va du $\mathrm{X}^{\mathrm{e}}$ au XII ${ }^{\mathrm{e}}$ siècle a vu l'Europe se faire et la culture européenne se former, comme l'a rappelé Georges Duby ${ }^{21}$. Furent alors expérimentés des pouvoirs et des rôles qui peu après se fixèrent, au moins en ce qui concerne les rapports entre les sexes. L'historiographie italienne s'accorde avec G. Duby pour reconnaître à la période du haut Moyen Age et du Moyen Age central une certaine participation des femmes à la vie publique, encore que limitée, et les travaux de G. Duby sur le XII siècle ont sans aucun doute étayé cette conviction. La femme noble du XII ${ }^{\mathrm{e}}$ siècle s'est vu concéder une position importante dans toute l'Europe centrale, Pologne et Bohême comprises. Beaucoup des contributions au fameux recueil de la Société Jean Bodin datant de 1962 l'avaient bien montré2 ${ }^{2}$. Les historiens s'accordent encore sur le fait qu'on ne peut en dire autant pour la période qui la suit, ces XIII ${ }^{e}$-XVIII ${ }^{e}$ siècles retenus comme cadre chronologique par les organisateurs de la XXI Semaine d'études de l'Institut international d'histoire économique F. Datini de Prato. En 1989, le thème en était « La femme dans l'économie » et G. Duby participa à la table-ronde qui conclut la rencontre ${ }^{23}$. Il commença par déclarer son inaptitude à conclure les travaux d'un colloque portant sur une période qui commençait précisément là où lui-même renonçait à s'occuper d'histoire des femmes. La période qu'il avait choisie, ce XIIe siècle qu'il privilégiait, ne permettait aucun sondage ou étude sur les femmes du peuple ou d'humble extraction. Ses déclarations laissaient entendre que c'était justement ces lacunes de la documentation qui l'avaient poussé à se concentrer sur les femmes de la classe dominante, les Dames du XII ${ }^{e}$ siècle.

Son intervention au colloque de 1989 lui donna pourtant l'occasion de dresser un rapide bilan en cinq points. Ils me paraissent dessiner un cadre où mener la confrontation entre les orientations de recherche les plus récentes et ses propres conceptions. Je les présenterai donc successivement en me référant aux travaux italiens qui ont avalisé ou contredit ses propositions. 


\section{Les femmes dans les échanges matrimoniaux}

mier point : Les femmes ont été considérées comme objets d'échange et instruments dans la conclusion des alliances et des paix. De là découle leur instrumentalisation, mais aussi la reconnaissance de leur valeur, que celle-ci fût liée à leur capacité procréatrice ou à la transmission de droits à leur descendance qu'elles lui ont assurée pendant une certaine période.

Second point : C'est de la reconnaissance de cette valeur que naît, dans le souci d'éviter l'éparpillement des patrimoines, l'assignation de dots aux femmes en échange de la renonciation qui leur est imposée à toute prétention sur le patrimoine.

Le premier point est peut-être le plus important. Il concerne le rapport des femmes au pouvoir et conduit à réfléchir non seulement sur les exemples d'autorité politique exercée par quelques femmes - Aliénor d'Aquitaine, Mathilde de Canossa ou leurs émules - mais sur un pouvoir féminin plus répandu quoique moins célébré, et bien réel pourtant. La femme qui, cédée par sa famille de naissance, assurait la paix sociale, celle qui jetait un pont entre deux lignages et dont les maternités assuraient une descendance légitime, celle-là détenait un pouvoir effectif. En réalité, le thème inclut l'institution du mariage tout entière, il touche en particulier aux droits que la femme conservait lorsqu'elle contractait un lien matrimonial et à ceux que son mari acquérait sur elle.

21 L'historiographie italienne s'est intéressée à ces thèmes comme le montre la veine nourrie de travaux qui lui est consacrée, jusqu'à la toute récente Storia del matrimonio ${ }^{24}$. Mais il faut d'abord renvoyer aux essais de M. Bellomo ou de G. Vismara ${ }^{25}$ et à l'étude de M. T. Guerra Medici parue en 1986 qui porte sur les droits des femmes pendant le haut Moyen $\mathrm{Age}^{26}$. Ce travail, qui se situe dans une solide tradition historiographique, est le plus complet sur la position de la femme dans la famille et la société du haut Moyen Âge. Certes, il recourt surtout à ces sources juridiques que G. Duby recommandait de manier avec prudence. M. T. Guerra Medici ne renvoie pas souvent à G. Duby et mentionne bien plus fréquemment M. Bellomo, mais G. Duby fait évidemment partie de ses lectures de base. Or, à l'en croire, c'est justement au XII siècle qu'on voit se restreindre la sphère d'influence de ces « dames » que le talent narratif de G. Duby nous a rendues familières.

Parmi ceux qui ont enquêté sur la position de la femme dans la famille et la société jusqu'au XII ${ }^{e}$ siècle, il faut mentionner les noms de B. Vetere, pour une étude contenue dans un recueil de 1986, de P. M. Arcari et surtout de Cinzio Violante; aucun d'entre eux, cependant, n'est historien du droit. L'importante étude, déjà ancienne, de P. M. Arcari portait plus particulièrement sur les idées et les sentiments politiques prévalant dans le haut Moyen Age, mais il a prêté attention à la manière dont on considérait les femmes ${ }^{27}$. C. Violante, quant à lui, participa en 1974 au colloque organisé par G. Duby et J. Le Goff sur Famille et parenté et y donna une contribution importante ${ }^{28}$. Il y enquête sur le rapport entre patrimoine familial et descendance et il note comment au XIe siècle les femmes, spécialement en Lombardie, disposaient d'une notable autonomie. Il souligne lui aussi la nécessité pour les familles désireuses de protéger leur patrimoine d'en empêcher la dispersion par l'entremise des femmes; ce qui se traduit par l'exclusion des filles de l'héritage paternel et, à titre de compensation, par l'institution d'une dot à leur bénéfice. La restriction de la famille, son évolution vers la famille conjugale constituent, selon lui, un héritage que cette période du Moyen Âge va léguer 
aux siècles immédiatement postérieurs. Mais on assiste aussi, en sens contraire à cette tendance, à la formation de vastes consorterie, groupements de plusieurs branches dérivant d'une souche unique qui s'identifient par le port d'un même nom. C'est à l'intérieur de ces vastes réseaux que les femmes devaient finir par être lentement englouties.

D'autres études, celles par exemple de M. Montanari ${ }^{29}$, P. Galetti ${ }^{30}$ et plus récemment S. Bernardi, quoique pour une époque plus tardive ${ }^{31}$, nous permettent de suivre l'évolution qui fait apparaître des femmes aux côtés des hommes dans les transactions foncières. Contrats agraires, emphytéoses, livelli et donations de biens immeubles sont tous des institutions juridiques où se révèle pendant le haut Moyen Age l'implication des femmes à l'égal des hommes. La femme agisssait à la première personne, ou aux côtés de son mari ou d'un parent, surtout dans les zones de tradition romaine. Sur les liens entre les femmes et le patrimoine, la lignée de leurs descendants ou la dot, nous disposons de quelques études récentes, relatives surtout au bas Moyen Âge : celles rassemblées par C. Klapisch-Zuber en divers recueils ${ }^{32}$, l'essai d'Isabelle Chabot-Pirillo sur les ressources et droits patrimoniaux des femmes au travail ${ }^{33}$, et les contributions à l'Histoire du mariage plus haut mentionnée, celles en particulier de Diane O. Hughes et de Lorenzo Fabbri ${ }^{34}$. D. O. Hughes observe qu'au XII siècle les échanges afférents au mariage ont subi une profonde transformation largement liée à l'institution dotale, mais que le mariage dotal ne s'affirme pas avec la même force dans toute la péninsule italienne ni dans tous les milieux sociaux. Entre $\mathrm{XII}^{e}$ et $\mathrm{XIII}^{\mathrm{e}}$ siècle, l'Église intervint aussi de façon appuyée en faveur de l'indissolubilité du lien conjugal, une orientation qui a progressivement changé la politique matrimoniale de l'aristocratie dans le nord de l'Europe, comme l'a montré G. Duby, repris sur ce point par D. O. Hughes. I. Chabot aborde aussi, pour la fin du Moyen Âge, le thème de l'exclusion des filles de la succession agnatique et celui de la dot comme compensation monétarisée concédée aux filles en échange de leur renonciation à l'héritage paternel, un phénomène qui démarre dans la période étudiée par G. Duby. Mais, en quelques siècles, l'inégalité entre filles et garçons face à l'héritage s'est accentuée ; une césure nette divise haut et bas Moyen Âge, non seulement à cet égard, mais en ce qui concerne la condition ainsi que la considération accordée à la femme, et c'est dans la dernière période que les femmes semblent souffrir des plus grandes restrictions.

Le thème de la capacité procréatrice, celui du pouvoir qui dérivait de la maternité pour les femmes, ont été moins abordés que celui du mariage. Le volume consacré à ces problèmes, qu'a édité Marina D'Amelia dans la série « Histoire des Italiennes ", part de la fin du Moyen Âge ${ }^{35}$. Il nous est resté, cependant, un témoignage, d'autant plus précieux qu'il est plus rare, sur la sensibilité et l'expérience d'une mère du IX ${ }^{\mathrm{e}}$ siècle, le Liber manualis de Dhuoda dont s'est occupé Franco Cardini il y a quelques années ${ }^{36}$. Les fils étaient très tôt éloignés de leur mère, et la séparation laissait chez les uns et les autres une nostalgie aiguë, origine fréquente d'une peur des femmes chez ces hommes ayant si peu connu la première femme qui compte dans la vie de chacun, la mère. Dhuoda était une femme d'une culture exceptionnelle pour son temps, et son œuvre révèle, selon $\mathrm{F}$. Cardini, à travers la culture de l'époque, une véritable religion de la paternité, une éthique de la fidélité, un ensemble de valeurs qui seront celles du monde féodal et chevaleresque, des connaissances numérologiques et symboliques spécifiques de la période, ainsi qu'une fervente confiance dans la prière, vue peut-être comme 
invitation à fuir le monde environnant, enfin la nostalgie dévorante de la relation maternelle trop tôt interrompue.

Les mères médiévales, celles en particulier qui appartiennent à d'autres milieux que Dhuoda, sont reflétées à loisir dans le miroir précieux, bien que déformant, de la littérature consacrée aux péchés. Dans le troisième volume des Dames $d u X{ }^{e}$ siècle, traduit en italien sous le titre "Les péchés des femmes au Moyen Âge", G. Duby s'occupe brièvement du dernier des pénitentiels médiévaux, le livre XIX du Décret de Burchard de Worms composé au début $\mathrm{du}_{\mathrm{XI}}{ }^{\mathrm{e}}$ siècle. Les mères qui soignent leurs enfants par des moyens que l'Église juge illicites parce que trop entachés de superstitions, celles qui, confinées au seul commerce des femmes et des enfants transfèrent sur ces derniers leur besoin de satisfaction sexuelle, les femmes qui refusent la perspective d'une maternité et se décident à supprimer leur fruit ou cherchent un moyen d'éviter une grossesse, telles sont les protagonistes de la littérature pénitentielle du haut Moyen Âge. Les livres pénitentiels parlent des usages et de la mentalité des pécheurs, mais ils témoignent aussi des peurs et des projets de ceux qui administraient la pénitence. Raoul Manselli est, en Italie, l'un de ceux qui ont étudié cette littérature afin de mieux comprendre les superstitions et la sexualitée ${ }^{37}$. D'autres, par la suite, ont aussi utilisé cette source pour connaître les rôles tenus par les femmes et ceux qu'on leur assignait ${ }^{38}$.

\section{Femmes oisives et femmes actives}

Troisième point des conclusions de G. Duby à Prato : À l'intérieur de la maison, la femme exerce un pouvoir authentique, spécialement dans la chambre des dames des demeures aristocratiques. La maison est le royaume de la dame, mais aussi son ghetto. Elle en sort ou pour entrer dans le circuit des échanges matrimoniaux ou pour servir de mannequin exhibant la richesse et le pouvoir de son mari. Dans la domus, où son autorité s'étend sur la domesticité et sur toutes les femmes qui y circulent, l'économie repose essentiellement sur elle. En l'absence de son époux, elle exerce aussi une partie du pouvoir qui revient au dominus.

Le quatrième point est lié au précédent. Même les femmes riches ne doivent jamais rester oisives: on considère alors l'exercice d'activités pratiques comme le meilleur antidote aux inclinations naturellement mauvaises des femmes. Toutes, de la plus noble à la plus humble, se consacrent à la production textile, participant de la sorte dans une mesure non négligeable à l'économie, en particulier à la production ostentatoire.

Si l'on combine ces troisième et quatrième points, le discours se déplace des péchés aux vices des femmes, argument du dernier volume de G. Duby. Selon les hommes d'Église des X $\mathrm{X}^{\mathrm{e}} \mathrm{XIII}{ }^{\mathrm{e}}$ siècles - et, il faut le reconnaître, bien plus encore selon ceux du bas Moyen Âge - les femmes sont éminemment enclines au vice et c'est pourquoi elles sont gardées à la maison et jamais laissées oisives. Ce thème de la "femme gardée » a été affronté par Carla Casagrande dans l'essai qu'elle a donné à l'Histoire des femmes en Occident ${ }^{39}$. Elle avait publié quelques années plus tôt les textes de sermons expressément adressés à des publics féminins par les prédicateurs Humbert de Romans, Gilbert de Tournai et Étienne de Bourbon ${ }^{40}$. Dans ces prêches, il est, pour la première fois, prêté attention à la condition sociale des femmes, qui ne sont plus exclusivement vues comme vierges, mères ou veuves ${ }^{41}$. Car les prédicateurs affrontent un public de femmes vivant non plus dans des bourgades isolées mais dans les villes, au contact de beaucoup de gens, et ces 
femmes sont soumises à des sollicitations constamment renouvelées: les religieux prennent conscience de la nécessité de bien cibler leurs interventions en évitant d'être vagues. À dater du XIII ${ }^{e}$ siècle, ce ne sont pas seulement les prédicateurs, mais les intellectuels laïcs qui, dans une intention pédagogique, s'occupent avec ardeur de former la bonne épouse ou la jeune fille bien élevée ${ }^{42}$.

La dame vivant au château, seul type féminin étudié en profondeur par G. Duby, sortait rarement, mais on ne peut non plus dire qu'elle ne se déplaçait pas du tout. Mathilde et Adélaïde ont mené d'innombrables et épuisantes expéditions politiques ou des pélerinages religieux, tous voyages ou sorties qui exigeaient le respect de règles et d'un apparat bien précis. Mais la perspective du rapport entre le dehors et le dedans change radicalement lorsqu'on aborde le bas Moyen Âge et que l'on considère la vie des citadines $^{43}$. G. Duby avait relié ce thème, dans le quatrième point de ses conclusions, à l'engagement des dames dans la production de textiles souvent précieux, de ces draps qu'elles endossaient elles-mêmes dans les grandes occasions où elles avaient à représenter le pouvoir de leur famille. Les riches habits dans lesquels elles apparaissaient alors leur conféraient une identité familiale, non pas personnelle; ils les rendaient certes visibles, mais en qualité de femmes ou de filles des hommes qui gardaient le contrôle pratique et symbolique de leur apparence esthétique. Or, si les changements furent nombreux et importants au bas Moyen Âge, la fonction d'emblème de la richesse ou des privilèges familiaux qui revient aux femmes lorsque leurs hommes exposent robes et joyaux sur leurs corps, appartient toutefois autant à la dame du XII ${ }^{e}$ siècle qu'aux citadines des derniers siècles du Moyen Âge ${ }^{44}$.

30 À ce quatrième point se rapporte, de façon plus générale, le thème du travail des femmes, sur lequel on dispose maintenant de deux recueils récents ${ }^{45}$. À la différence du second, le premier est exclusivement dédié à l'époque médiévale. Certains de ses essais traitent de questions évoquées par G. Duby, telle la contribution de B. Andreolli qui discute la constance du travail féminin et la part qu'elles occupent, dames comprises, dans la production textile. Non seulement ce travail a une réelle importance économique, mais il tient les femmes occupées à des activités compatibles avec leurs autres obligations, les grossesses en premier lieu. On peut donc qualifier ces activités peu visibles d'interstitielles ou complémentaires ${ }^{46}$.

31 Les contributions de Gabriella Piccinni et d'Isabelle Chabot au volume Il lavoro delle donne coordonné par Angela Groppi ${ }^{47}$ et une autre d'Isabelle Chabot, au colloque de Prato de $1989^{48}$, touchent aux sujets évoqués par G. Duby, encore que les contributions d'I. Chabot se déplacent vers une époque plus tardive, les deux derniers siècles du Moyen Age. Comme le montre l'essai de G. Piccinni, qui renvoie expressément à l'intervention de G. Duby à Prato, le travail féminin « a joui d'une considération et d'un espace plus grands dans le haut Moyen Age qu'à la fin de la période et à l'époque moderne. L'impression de cette réduction naît de la constatation que les femmes de l'aristocratie ont exercé à l'époque féodale des rôles importants ${ }^{49}$ ». Pour nuancer cette appréciation et juger de la place économique des femmes en dehors des milieux aristocratiques, nous signalerons aussi le tableau, si partiel soit-il, de la situation sociale et juridique de la femme à Plaisance entre XII ${ }^{e}$ et XIII ${ }^{e}$ siècles. Il met en lumière la présence féminine dans les activités artisanales, ainsi que l'égalité avec les hommes devant les magistrats de la ville $e^{50}$. Il en allait de même à Gênes ${ }^{51}$ et vraisemblablement dans beaucoup d'autres villes où elles étaient surtout engagées dans la production 
artisanale. Entre XIII ${ }^{e}$ et XIVe siècles, diverses raisons d'ordre politique et économique ont fait que leur condition s'est presque partout aggravée ${ }^{52}$.

Dans sa contribution à l'Histoire des femmes en Occident intitulée « La femme imaginée " ${ }^{53}$ - et faut-il rappeler que l'histoire par les images fut l'une des passions de G. Duby ${ }^{54}$ ? Chiara Frugoni part des représentations de la femme inspirées par l'Église, pour aborder le «droit à l'image » conquis au bas Moyen Âge par les femmes qui vivaient et travaillaient dans les villes. Cette approche amène l'auteur à nuancer la thèse d'une dégradation de la situation des femmes lorsqu'on passe du Moyen Age central à la fin de la période. Cette conquête du droit à apparaître concerne du reste non seulement les femmes travaillant dans les boutiques ou les hospices, mais aussi celles représentées dans un paysage rural ${ }^{55}$. Suivre la piste du travail féminin est donc l'une des manières de saisir le rôle social des femmes, surtout au bas Moyen Âge, alors que, pour la période précédente, il est peut-être plus profitable de considérer l'apparition des femmes dans les documents en tant que sujets dotés de droits analogues à ceux des hommes.

\section{Une reconsidération des femmes}

33 Le cinquième point de G. Duby reprenait, à Prato, ces problèmes. Sans mentionner le type de sources tout juste évoqué, il y affirmait que la femme occupait une position de relief dans la société féodale. La société féodale connaissait non pas la marginalisation, mais la ségrégation des femmes, dans le cadre d'une structure sociale fondée sur la domus, à l'intérieur de laquelle se distinguaient deux domaines, l'un masculin, l'autre féminin. Si les sources nous donnent parfois l'impression d'une marginalité féminine, c'est que leurs auteurs étaient des hommes qui, en théorisant l'infériorité des femmes, exorcisaient la peur qu'elles leur inspiraient.

Le $\mathrm{XII}^{\mathrm{e}}$ siècle a suscité dans divers milieux et pour des raisons d'ordre varié une reconsidération de la figure féminine et les travaux de Jean Leclercq ont mis en lumière la contribution monastique à cette réappréciation ${ }^{56}$. Peut-on pour autant parler de promotion de la condition féminine à l'époque féodale ${ }^{57}$ ? Et l'amour courtois a-t-il joué un rôle dans cette promotion en accordant à la femme un réel pouvoir?

Le rapport entre le rôle de la femme dans le modèle littéraire courtois et celui qu'elle tient dans la vie réelle demeure obscur et il semble tout aussi difficile de mesurer l'intensité des rôles féminins dans le jeu même de l'amour courtois. Peut-être doit-on partir d'une question : qui a créé le modèle de relation amoureuse connu sous le nom d'«amour courtois ", et dans quel but? D'aucuns soutiennent que ses inventeurs doivent être identifiés dans le milieu social des grands feudataires, d'autres le situent dans celui de la petite noblesse ${ }^{58}$. Rien pourtant - et G. Duby l'a dit - ne permet de croire qu'il ait été une invention des femmes ${ }^{59}$. Il semble solidement établi que l'amour courtois reflète une situation conflictuelle entre le vassal et son seigneur : un conflit, autrement dit, entre des couches différentes de la société féodale, et sur ce point on connaît la thèse d'E. Köhler, pour qui la chevalerie et la petite noblesse aspiraient à s'élever en s'intégrant à l'aristocratie ${ }^{60}$. Mais il reflète aussi un conflit de générations, qui oppose fils et pères pour l'amour de la dame. La reine ou la dame attire parce qu'elle est la femme du roi ou du seigneur, ou tout au moins d'un personnage de rang supérieur à celui de l'amant. Quoi qu'il en soit, l'amour courtois valorise le pouvoir que représente une dame, non pas la femme en soi. Et même si les discours portent uniquement sur la dame, il reste que cette femme assume dans une relation de ce type 
une fonction de représentation du pouvoir tout à fait originale. C'est pour la possession de la reine que luttent roi et chevalier. Et si, pour arriver à la souveraineté, il faut conquérir la reine, il en découle que c'est la reine qui confère la souveraineté. Pures abstractions, jeux de cour, questions de nature anthropologique, dira-t-on. C'est vrai, mais il ne s'agit pas que de cela. Car l'histoire d'Henri Plantagenet ou de Guillaume le Maréchal et de leurs heureux mariages tels que G. Duby les a reconstitués, nous fait glisser du rêve à la réalité.

La femme par qui passe l'accès au pouvoir est-elle pour autant une figure royale ? De ce rapport entre personnes qui, en réalité, est un défi entre couches sociales, un jeu de pouvoir aux règles fort complexes, deux philologues romans, Andrea Fassò et Mario Mancini, ont traité à propos du problème de la dame au XII ${ }^{e}$ siècle ${ }^{61}$. C'est A. Fassò qui a soulevé la question de la relation entre la domna des troubadours et la fée celtique. Notre figure de dame quasi surnaturelle, digne de la plus grande vénération, dispensatrice de richesses, de force et de pouvoir, ne s'est-elle pas modelée sur celle de la fée ${ }^{62}$ ? Peut-être G. Duby aurait-il accepté l'hypothèse, puisqu'il a souvent souligné la dimension onirique présente dans l'amour courtois et noté comment celui-ci s'était développé dans un champ imaginaire et ludique. Cela dit, lorsqu'il parlait de la domina placée au centre de ce jeu, il mettait l'accent sur les aspects historiques qui en faisaient l'épouse du seigneur, la puissante patronne d'une maison, la responsable de sa gestion. Il est possible qu'en rêvant d'une fée, les hommes aient renforcé le pouvoir des femmes de leur milieu.

Dans les cours seigneuriales où l'on assiégeait la femme du maitre, s'est déclenché un processus de civilisation fondé sur le respect de règles précises et sur la théorisation du report de la satisfaction des pulsions jusque sur le terrain très particulier de l'amour. À partir du XII ${ }^{e}$ siècle, les témoignages se multiplient d'une discipline sociale croissante ; elle implique aussi des milieux étrangers à la cour seigneuriale et entrâne la production de codes de comportement qu'on peut ramener à un courant clérical ou courtois $^{63}$. C'est dans la société provençale que naît le premier projet séculier d'éducation. Il exalte diverses qualités féminines, cette beauté en particulier que l'Église s'ingénie au contraire à mortifier. Aux femmes nobles et de haut rang s'adressent des recommandations et des enseignements faisant partie d'une production littéraire qui, certes, tourne toute entière autour de la domina; elle n'en affirme pas moins des valeurs et suscite des comportements montrant qu'une autre relation avec les femmes que celle définie par les clercs est possible, au moins dans un milieu social limité. Peut-être les troubadours rêvaient-ils d'une fée, mais c'est aux femmes bien réelles que les traités pédagogiques conçus dans les milieux de cour dictaient leurs enseignements sur l'attention à prêter au corps ou sur la conduite à tenir ${ }^{64}$.

Le lien entre la conception que lettrés de cour ou théologiens se font des femmes ${ }^{65}$ et les occasions réelles qu'avaient celles-ci de s'affirmer dans la société du XII ${ }^{\mathrm{e}}$ siècle reste crucial. Si derrière la conception des troubadours nous découvrons des strates multiples d'influences culturelles, nous en dégageons tout autant derrière le discours des théologiens du XII e siècle qui parlent des femmes. Ce discours s'adresse à un type féminin abstrait, qui pouvait être contredit par les femmes réelles et apparaitre bien éloigné de leurs figures concrètes. Aussi les conclusions auxquelles nous mènent les études sur les conceptions médiévales de la femme et ses rôles effectifs divergent-elles sensiblement. 

ont fondé leurs arguments sur toute une tradition. Pour eux, la pensée d'Isidore de Séville, d'Ambroise et d'Augustin comptait bien plus que toute expérience concrète se déroulant sous leurs yeux. Cela, qui était inévitable, a tout aussi inévitablement créé un filtre engendrant des déformations. C'est pour esquiver un tel risque que G. Duby a choisi d'étudier les femmes réelles, de vérifier de quel pouvoir elles ont réussi à s'emparer, de considérer les phases de l'histoire où s'est manifestée le plus clairement la distance entre le modèle théorique et les comportements réels, entre par exemple les raisonnements d'Yves de Chartres et ceux des pères qui arrangeaient le mariage de leurs filles ${ }^{66}$. On saisit mieux les écarts, les rapprochements ou les conditionnements réciproques lorsqu'on argumente, comme l'a fait $\mathrm{G}$. Duby, sur l'institution particulière du mariage, le plus élémentaire des rapports sociaux de $\operatorname{sexe}^{67}$. C'est justement ce thème des liens matrimoniaux et familiaux - qu'ils soient affectifs, économiques, stratégiques ou de pouvoir - qui a constitué l'un des terrains d'élection de $l^{1}$ historiographie italienne dans les récentes années ${ }^{68}$, parce qu'il se prête excellemment à la confrontation de la théorie et de la pratique, des mots et des choses.

Si la recommandation que fit à G. Duby son professeur de philosophie : choisir les choses plutôt que les mots, a effectivement joué un rôle dans sa conversion aux études historiques, nous ne pouvons que lui en être reconnaissants, en particulier quant à l'histoire des femmes ${ }^{69}$. Car c'est bien la recherche de l'historien Duby qui a démontré que les mots sont aussi des choses, que l'histoire matérielle et celle des mentalités ne peuvent être disjointes sans entraîner de graves distorsions compromettant les résultats acquis, en Italie et ailleurs, dans la difficile reconstitution de l'histoire des femmes au Moyen Âge - une histoire qui est comme l'épopée féminine de notre Far West, aurait peut-être dit G. Duby, à la recherche de notre identité d'hommes et de femmes, et pourquoi pas, d'un espace où rêver.

\section{BIBLIOGRAPHIE}

Altrove...

1998 Altrove. Viaggi di donne dall'antichità al Novecento. Atti del seminario di Firenze, 20-21 febbraio 1998, à paraître.

ANDREOLLI Bruno

1991, « Tra podere e gineceo. Il lavoro delle donne nelle grandi aziende agrarie dell'alto medioevo », dans MUZZARELLI, ANDREOLLI et GALETTI 1991, pp. 29-40.

ARCARI Paola Maria

1968 Idee e sentimenti politici dell'alto Medioevo, Milan, Giuffrè.

BELLOMO Manlio

1961 Ricerche sui rapporti patrimoniali tra coniugi. Contributo alla storia della famiglia medievale, Milan, Giuffrè.

Clio. Femmes, Genre, Histoire, 8 | 1998 
1965 « Dote. Diritto intermedio », dans Enciclopedia del diritto, vol. XIV, Milan, Giuffrè.

1970 La condizione giuridica della donna in Italia. Vicende antiche e moderne, Turin, ERI.

BERNARDI Simonetta

1996 « La donna nel mondo comunale fra vita familiare e gestione del patrimonio (esempi da documenti marchigiani dei secc. XIII e XIV », dans G. Pagliano (dir.), La presenza dimenticata. Il femminile nell'Italia moderna fra storia, letteratura, filosofia, Milan, Franco Angeli, pp. 9-27.

BERTINI Ferruccio et al.

1989 Medioevo al femminile, Rome-Bari, Laterza.

BIANCO Marinella

1995 « Le classificazioni femminili nella mentalità medievale (secc. XII-XVI) », Nuova rivista storica, II, pp. 261-274.

CALVI Giulia (dir.)

1992 Barocco al femminile, Rome-Bari, Laterza.

CANTARELLA Glauco Maria

1997 Principi e corti. L’Europa del XII secolo, Turin, Einaudi.

CARDINI Franco

1979 Magia, stregoneria, superstizioni nell'Occidente medievale, Florence, La Nuova Italia Editrice.

1989 « Dhuoda, la madre », dans BERTINI 1989, pp. 41-62.

CASAGRANDE Carla

1978 (ed.) Prediche alle donne del secolo XIII. Testi di Umberto da Romans, Gilberto da Tournai, Stefano di Borbone, Milan, Bompiani.

1991 « La femme gardée », dans DUBY et PERROT 1991, pp. 83-116.

CASAGRANDE Carla et VECCHIO Silvana

1991 Les péchés de langue, Paris, Ed. du CERF (éd. ital. 1987)

CAVACIOCCHI Simonetta (éd.)

1990 La donna nell'economia, secc. XIII-XVIII. Atti della XXI. Settimana di studi dell'Istituto internaz. "F. Datini" di Prato, Florence.

CHABOT Isabelle

1990 « La reconnaissance du travail des femmes dans la Florence du bas Moyen Âge : contexte idéologique et réalité », dans La donna nell'economia... 1990, pp. 563-576.

1994 « "La sposa in nero". La ritualizzazione del lutto delle vedove fiorentine (secoli XIV-XV) », Quaderni storici, 86, pp. 421-462.

1996 « Risorse e diritti patrimoniali », dans GROPPI 1996, pp. 47-70.

COLLINA Beatrice

1996 « L'esemplarità delle donne illustri fra Umanesimo e Controriforma », dans G. Zarri (dir.), Donna, disciplina, creanza cristiana dal XV al XVII secolo. Studi e testi a stampa, Rome, Edizioni di Storia e Letteratura, pp. 103-119. 
1997 « Donna illustre e guerriera di Dio : Matilde nella letteratura fra Tre e Cinquecento », dans Matilde di Canossa nelle culture europee del II. millennio, Convegno internaz. di studi, Reggio Emilia, 25-27 sett. 1997 (à paraître).

La Contessa Adelaide...

1992 La contessa Adelaide e la società del secolo XI. Atti del convegno di Susa, 14-16 nov. 1991, Segusium. Società di ricerche e studi valsusini, XXIX, Susa, Deputazione Subalpina di storia patria.

CORSI Dinora

1990 « Dal sacrificio al maleficio. La donna e il sacro nell'eresia e nella stregoneria », Quaderni medievali, 50, pp. 8-62.

D’AMELIA Marina (dir.)

1997a Storia della maternità, Rome-Bari, Laterza [coll. "Storia delle Italiane].

$1997 b$ « La presenza delle madri nell'Italia medievale e moderna », dans D'AMELIA, 1997a, pp. 3-52.

DE GIORGIO Michela et KLAPISCH-ZUBER Christiane (dir.)

1996 Storia del matrimonio, Rome-Bari, Laterza [coll. "Storia delle Italiane"].

DE MATTEIS Maria Consiglia

1981 (dir.) Idee sulla donna nel Medioevo : fonti e aspetti giuridici, antropologici, religiosi, sociali e letterari della condizione femminile, Bologna, Pàtron Editore.

1992 « Ruoli femminili della politica nel secolo XI », dans La contessa Adelaide 1992, pp. 27-41.

La donna nell'economia...

1990 La donna nell'economia, secc. XIII-XVIII, Atti della XXI. Settimana di Studi, 1989, Florence, Le Monnier [Istituto Internazionale di storia economica "F. Datini", Prato, ser. II, n 21].

DRONKE Peter

1984 Women writers of the Middle Ages. A critical study of texts from Perpetua to Marguerite Porete, Cambridge, Cambridge University Press (tr. ital. 1986).

DUBY Georges

1977 « Le mariage dans la société du haut Moyen Age », dans Il matrimonio..., pp. 15-39. Réimpr. dans Duby 1988, pp. 9-33 (tr. ital. 1988).

1978 Mediaeval Marriage. Two models from Twelfth Century France, Baltimore-Londres, The Johns Hopkins University Press (tr. ital. 1981).

1981 Le Chevalier, la femme et le prêtre. Le mariage dans la France féodale, Paris, Hachette (tr. ital. 1982).

1988 Mâle Moyen Age. De l'amour et autres essais, Paris, Flammarion (tr. ital., 1988).

1991 « Le modèle courtois », dans DUBY et PERROT 1991, pp. 261-276.

1996 Dames du XIIe siècle, Paris, Gallimard, 1995-1996, 3 vol. (Est plus particulièrment cité ici le vol. III : Ève et les prêtres, 1996 ; tr. ital. 1997)).

DUBY Georges et GEREMEK Bronislaw

1992 Passions communes. Entretien avec P. Sainteny, Paris, Ed. du Seuil (tr. ital. 1993). 
DUBY Georges et LE GOFF Jacques (dir.)

1977 Famille et parenté dans l'Occident médiéval. Actes du Colloque de Paris, 6-8 juin 1974, Rome, Ecole Française de Rome (tr. ital. 1981).

DUBY Georges et PERROT Michelle (dir.)

1991 Histoire des femmes en Occident, II : Le Moyen Age (sous la dir. de C. Klapisch-Zuber), Paris, Plon (l'édition italienne est parue en 1990 chez Laterza).

1992 Images de femmes, Paris, Plon.

FABBRI Lorenzo

1996 « Trattatistica e pratica dell'alleanza matrimoniale », dans DE GIORGIO et KLAPISCH-ZUBER, 1996, pp. 91-117.

FASSÒ Andrea

1989 « La lotta col re-padre e il sogno della sovranità : gli eroi di Chretien de Troyes », dans L'immagine riflessa. Rivista di sociologia dei testi, XII, pp. 55-90.

1997 « La diffrazione e le fate. (Guglielmo IX, Ben vueill que sapchon li pluzor) », dans Atti del V Congresso nazionale della Società italiana di filologia romanza (Rome, 23-25 ott. 1997), à paraître.

FAZIO Ida

1995 « Relazione generale alla prima sezione del I Congresso delle Storiche italiane (Rimini, 9 giugno 1995) », Agenda, 15, p. 29-38.

FRUGONI Chiara

1977 « L'iconografia del matrimonio e delle coppie nel Medioevo », dans Il matrimonio... 1977, pp. 901-963.

1991 « La femme imaginée », dans DUBY et PERROT 1991, pp. 357-439.

FUMAGALLI Vito

1992 « Adelaide e Matilde, due protagoniste del potere medievale », dans La contessa Adelaide 1992, pp. 243-257.

1996 Matilde di Canossa. Potenza e solitudine di una donna del Medioevo, Bologne. Il Mulino.

GALETTI Paola

1991 « La donna contadina. Figure femminili nella contrattualistica agraria italiana dell'alto medioevo », dans MUZZARELLI, ANDREOLLI et GALETTI 1991, pp. 41-54.

GATTI Daniela

1991 «Curatrici e streghe nell'Europa dell'alto medioevo », dans MUZZARELLI, ANDREOLLI et GALETTI 1991 , pp 127-140.

GIALLONGO Angela

1981 Il galateo e la donna nel Medioevo, Rimini, Maggioli.

GIORDANO Oronzo

1979 Religiosità popolare nell'alto Medioevo, Bari, Adriatica.

GOLINELLI Paolo

1991 Matilde e i Canossa nel cuore del Medioevo, Milan, Camunia. 
GRECI Roberto

1996 « Donne e corporazioni : la fluidità di un rapporto », dans GROPPI 1996, pp. 71-91.

GROPPI Angela (dir.)

1996 Il lavoro delle donne, Rome-Bari, Laterza [coll. "Storia delle Italiane"].

GUERRA MEDICI Maria Teresa

1986 I diritti delle donne nella società altomedievale, Naples [Ius nostrum. Studi e testi pubblicati dall'Istituto di storia del diritto italiano dell’Università di Roma, 2e série, 4].

HUGHES Diane Owen

1979 « Ideali domestici e comportamento sociale : testimonianze della Genova medievale ", dans C. E. Rosenberg (dir.), La famiglia nella storia. Comportamenti sociali e ideali domestici, Turin, Einaudi, pp. 147-183.

1987 « Invisible Madonnas? The Italian historiographical tradition and the women in medieval Italy », dans S. M. Stuard (dir.), Women in medieval History and Historiography, Philadelphia, University of Pennsylvania Press, pp. 25-57.

1996 « Il matrimonio nell'Italia medievale », dans DE GIORGIO et KLAPISCH-ZUBER 1996, pp. 5-61.

\section{KLAPISCH-ZUBER Christiane}

1988 La famiglia e le donne nel Rinascimento a Firenze, Rome-Bari, Laterza.

1990 La maison et le nom. tratégies et rituels dans l'Italie de la Renaissance, Paris, Ed. de l'EHESS.

1996 «Introduction », dans DE GIORGIO et KLAPISCH-ZUBER 1996, pp. VII-XX.

KÖHLER Erich

1974 L'aventure chevaleresque. Idéal et réalité dans le roman courtois. Etude sur la forme des plus anciens poèmes d'Arthur et du Graal, Paris, Gallimard [« Bibliothèque des idées »](tr. ital. 1989).

LECLERCQ Jean

1982 « L'amour et le mariage vu par des clercs et des religieux spécialement au XIIe siècle », dans Love and Marriage in the Twelfth Century, Louvain [coll. « Mediaevalia Lovaniensia », série I, stud. VIII], pp. 102-115 (tr. ital. 1984).

MANCINI Mario (dir.)

1991 Il punto su : I trovatori, Rome-Bari, Laterza.

MANSELLI Raul

1976 « Simbolismo e magia nell'alto medioevo », dans Simboli e simbologia nell'Alto Medioevo, Atti della XXIIIa Settimana di studi sull'Alto Medioevo, Spolète, pp. 293-329

1977a « Vie familiale et éthique sexuelle dans les Pénitentiels », dans DUBY et LE GOFF, pp. 363-378.

$1977 \mathrm{~b}$ « Il matrimonio nei penitenziali » dans Il Matrimonio... 1977, pp. 287-315.

Il Matrimonio...

1977 Il matrimonio nella società altomedievale, Atti della XXIVa Settimana di studio del Centro italiano di studi sull'alto medioevo, Spolète.

MONTANARI Massimo 
1988 Contadini e città fra "Langobardia" e "Romania", Florence, Salimbeni.

MUZZARELLI Maria Giuseppina

1986 « Le donne e i bambini nei libri penitenziali : regole di condotta per una società in formazione », dans VETERE et RENZI 1986, pp. 143-192.

1994 Penitenze nel Medioevo. Uomini e modelli a confronto, Bologne, Pàtron.

1996 Gli inganni delle apparenze. Disciplina di vesti ed ornamenti alla fine del Medioevo, Turin, Scriptorium.

MUZZARELLI M. G., ANDREOLLI B. et GALETTI P. (dir.)

1991 Donne e lavoro nell'Italia medievale, Turin, Rosenberg \& Sellier.

NICCOLI Ottavia (dir.)

1991 Rinascimento al femminile, Rome-Bari, Laterza.

OLDONI Massimo

1992 « L'iconografia letteraria di Adelaide », dans La Contessa Adelaide 1992, pp. 215-229.

PICCINNI Gabriella

1985 « Le donne nella mezzadria toscana delle origini. Materiali per la definizione del ruolo femminile nelle campagne », Ricerche storiche, XV, pp. 127-182.

1990 «Considerazioni dall'Italia medievale », dans CAVACIOCCHI 1990, pp. 71-81.

1996 « Le donne nella vita economica, sociale e politica dell'Italia Medievale », dans GROPPI 1996, pp. 5-46.

Recueils...

1962 Recueils de la Société Jean Bodin pour l'histoire comparative des institutions, XII : La femme, Bruxelles.

ROMAGNOLI Daniela

1991 « La courtoisie dans la ville : un modèle complexe », dans D. Romagnoli (dir.), La Ville et la Cour. Des bonnes et des mauvaises manières, Paris, Fayard, pp. 25-88 (éd. ital. 1991).

1994 « "Disciplina est conversatio bona et honesta" : anima, corpo e società tra Ugo di San Vittore ed Erasmo da Rotterdam », dans P. Prodi (dir.), Disciplina dell'anima, disciplina del corpo e disciplina della società tra medioevo ed Età moderna, Bologne, Il Mulino [Annali dell'Istituto storico italo-germanico in Trento, Quaderno 40], pp. 507-537.

VECCHIO Silvana

1991 « La bonne épouse », dans DUBY et PERROT 1991, pp. 117-146.

VETERE Benedetto

1986 « La donna nella società e nella cultura medioevale. Temi e itinerari di ricerca », dans VETERE et RENZI, 1986, pp. 19-102.

VETERE Benedetto et RENZI Paolo (dir.)

1986 Profili di donne. Mito, immagine, realtà fra Medioevo ed Età contemporanea, Galatina, Congedo Editore.

VIOLANTE Cinzio 
1977 «Quelques caractéristiques des structures familiales en Lombardi, Emilie et Toscane aux XIe et XIIe siècles ", dans DUBY et LE GOFF, 1977, pp. 87-147.

VIOLANTE Cinzio et FRIED Johannes (dir.)

1993 Il secolo XI : una svolta ?, Bologne, Il Mulino [Annali dell'Istituto storico italo-germanico in Trento, Quaderno 35].

VISMARA Giulio

1977 « I rapporti patrimoniali tra i coniugi nell'alto medioevo », dans Il matrimonio... 1977, pp. 633-691.

\section{ZANINONI Anna}

1989 «"Foemina, domina, massara". Appunti sulla condizione socio-giuridica della donna a Piacenza tra XII e XIII secolo », Nuova rivista storica, LXXIII, pp. 181-190.

\section{NOTES}

1. Duby 1996 : III, 217.

2. Duby 1981. Cf. mon compte rendu dans Rivista di storia e letteratura religiosa, XIX, 2, 1983, p. 312-317.

3. Duby 1978.

4. Duby 1977 ; repris dans Duby 1988.

5. Ibidem : 17-18.

6. Fumagalli 1996 : 90. Donne nelle specchio del Medioevo est la traduction italienne du premier tome de Dames du XII ${ }^{e}$ siècle, intitulé dans l'édition française : Héloïse, Aliénor, Iseut et quelques autres (Paris, Gallimard, 1995).

7. Fumagalli 1992.

8. Benzone d'Alba, Ad Henricum IV Imperatorem Libri VII, éd. par K. Pertz, dans M.G.H., Scriptores in usum scholarum, $65(\mathrm{~V}, 9)$; cf. Oldoni 1992. Dans le roman français, l'image correspondante est en revanche celle d'un cerf, souvent blanc, qu'il est difficile de chasser : cf. Fassò 1989.

9. Cantarella 1997 : 84-91, en part. 86. Sur Mathilde cf. aussi Golinelli 1991.

10. Hughes $1987: 29$. Cf. aussi l'introduction de Niccoli 1991 : V-XXVI, en part. VIII-XII.

11. Collina 1996 et 1997.

12. Bertini et al. 1989.

13. Même problématique dans Dronke 1984 .

14. Duby et Perrot $1991: 540$.

15. Bertini et al. 1989 : VI.

16. Niccoli 1991 : IX ; Calvi 1992.

17. Violante et Fried 1993.

18. Fumagalli 1992 : 243.

19. Fumagalli 1992 et 1996 ; Oldoni 1992.

20. D'autres participants au colloque de Susa dédié à Adélaïde ont repris ces idées avec des nuances diverses ; ainsi Maria Consiglia De Matteis, qui s'est occupée d'une autre femme ayant joué un rôle de premier plan dans la politique du $\mathrm{XI}^{\mathrm{e}}$ siècle. Il s'agit ici d'Agnès, fille du duc Guillaume V d'Aquitaine et de Poitou, épouse de Henri III de Franconie, devenue impératrice, et mère, enfin, de Henri VI. Cette Agnès aussi témoigne de la possibilité de renverser le cliché traditionnel qui voudrait voir dans la femme un être inerte et subordonné. Voir De Matteis 1992.

21. Duby et Geremek $1992: 112$.

22. Recueils 1962. 
23. Cavaciocchi $1990: 690-695$.

24. De Giorgio et Klapisch-Zuber 1996. Pour les travaux antérieurs, je renvoie aux bibliographies de cet ouvrage.

25. Bellomo 1961, 1965 et 1970 ; Vismara 1977.

26. Guerra Medici 1986.

27. Vetere 1986 ; Arcari 1968 : 474-521.

28. Violante 1977.

29. Montanari 1988

30. Galetti 1996.

31. Bernardi 1996

32. Klapisch-Zuber 1988 et 1991, en part. chap. IX, X et XII.

33. Chabot 1996 ; cf. Ead. 1994.

34. Hughes, 1996 ; Fabbri 1996.

35. D’Amelia 1997. Son essai propre ne concerne que partiellement l'époque médiévale ; ibidem: 3-52.

36. Cardini, 1989.

37. Manselli 1976, 1977A et 1977B.

38. Giordano 1979 ; Cardini 1979 ; Muzzarelli 1986 et 1994 ; Corsi 1990 ; Gatti 1991.

39. Casagrande 1990.

40. Casagrande 1978. Cf. aussi Casagrande et Vecchio 1991.

41. Bianco 1995.

42. Vecchio 1990

43. Cf. les actes du séminaire tenu à Florence les 20-21 février 1998, Altrove... 1998 (à paraître).

44. Muzzarelli 1996.

45. Muzzarelli, Andreolli et Galetti 1991 ; Groppi 1996.

46. Andreolli 1991.

47. Piccinni 1996 ; Chabot 1996 ; un troisième essai, celui de R. Greci, «Donne e corporazioni », traite de thèmes éloignés des intérêts de Duby ; Greci 1991.

48. Chabot 1990.

49. Piccinni 1996 ; Ead. 1985 et 1990.

50. Zaninoni 1989.

51. Hughes 1979.

52. Greci 1991.

53. Frugoni 1991. Le titre italien est « La donna nelle immagini, la donna immaginata ».

54. Duby et Perrot 1992.

55. Frugoni $1991: 367$.

56. Leclercq 1982.

57. Duby 1991.

58. Mancini 1991

59. Duby $1991: 264$.

60. Köhler 1974

61. Fassò 1989 ; Mancini 1991.

62. Fassò 1997.

63. Romagnoli 1991 et 1994.

64. Giallongo 1987 : 67-116.

65. De Matteis 1981.

66. Duby 1981.

67. Klapisch-Zuber 1996 : IX.

68. Fazio, $1995: 38$.

69. Duby et Geremek $1992: 11$. 


\section{RÉSUMÉS}

Dans ses études sur les dames au XII ${ }^{\mathrm{e}}$ siècle, G. Duby a défendu l'idée que la société féodale n'a pas marginalisé les femmes et qu'elles exerçaient un authentique pouvoir dans le cadre domestique. L'historiographie italienne qui a suivi des pistes analogues a plutôt mis l'accent sur le rôle politique que des femmes comme Mathilde de Canossa ou Adélaïde de Turin ont pu jouer au XI ${ }^{\mathrm{e}}$ siècle, en raison de conditions exceptionnelles propres à la période. Elle s'accorde avec G. Duby pour reconnaître dans le haut Moyen Âge et le Moyen Âge central une époque qui a consenti aux femmes une certaine participation. Il n'en va plus ainsi à la fin du Moyen Âge, quand elles ont souffert des plus fortes restrictions mises en lumière par les recherches récentes sur le travail et les droits féminins. Les travaux de G. Duby sur les femmes ont eu cet effet au moins sur l'historiographie italienne : faire reconnaître à ce champ d'enquête la pleine dignité que tant d'historiens lui accordent avec réticence.

In his studies of the women of the XII ${ }^{\text {th }}$ century, G. Duby asserted that women in feudal society were not marginalized and that they exerted a real power within their homes. Moving in the same directions, Italian historiography has stressed the political role that women such as Matilda of Canossa and Adelaide of Torino, were able to play, because of the exceptional circumstances of the $\mathrm{XI}^{\text {th }}$ century. Italian historiography agrees with G. Duby in crediting the early and high Middle Ages with allowing women some degree of participation. Things changed during the late Middle Ages when women's work and rights were more restricted, as recent studies have shown. Because of the attention G. Duby paid to women's history, many reluctant Italian scholars were forced to recognize the importance and dignity of this topic.

\section{AUTEUR}

\section{MARIA-GIUSEPPINA MUZZARELLI}

Maria-Giuseppina MUZZARELLI. Elle est chercheuse et enseigne à l'Université de Bologne. Ses recherches portent sur les mentalités et la société médiévales, en particulier sur la théorie et la pratique de la pénitence dans le haut Moyen Âge et le Moyen Âge central, sur quoi elle a publié en 1994 Penitenze nel Medioevo. Uomini e modelli a confronto (Bologne, Patron). Elle s'intéresse aussi aux rapports entre majorité chrétienne et minorité juive dans les villes de la fin du Moyen Âge, et elle a édité un volume sur ce thème, Verso l'epilogo di una convivenza. Gli ebrei a Bologna nel XVI secolo (Florence, Giuntina, 1996). Plusieurs de ses recherches ont été consacrées à l'histoire des femmes, au travail féminin ainsi qu'à diverses figures féminines comme Christine de Pizan ou Gracia Mendes. Elle travaille actuellement sur la réglementation du vêtement et des parures chez les législateurs et moralistes (Gli inganni delle apparenze, Turin, Scriptorium, 1996) et sur le rapport des personnes au vêtement. 